ELOHI

Peuples indigènes et environnement

5-6 | 2014

Ressources du vivant

Le végétal comme condition d'existence et d'incarnation : réflexions sur la notion de ressource chez les Yanesha en Haute Amazonie péruvienne

Céline Valadeau

2 OpenEdition

Journals

Édition électronique

URL : http://journals.openedition.org/elohi/721

DOI : 10.4000/elohi.721

ISSN : 2268-5243

Éditeur

Presses universitaires de Bordeaux

Édition imprimée

Date de publication : 1 janvier 2014

Pagination : 55-67

ISBN : 979-10-300-0021-4

ISSN : 2431-8175

Référence électronique

Céline Valadeau, «Le végétal comme condition d'existence et d'incarnation : réflexions sur la notion de ressource chez les Yanesha en Haute Amazonie péruvienne », ELOHI [En ligne], 5-6 | 2014, mis en ligne le 01 janvier 2015, consulté le 30 avril 2019. URL : http://journals.openedition.org/elohi/721 ; DOI : 10.4000/elohi.721 


\title{
Le végétal comme condition d'existence et d'incarnation: réflexions sur la notion de ressource chez les Yanesha en Haute Amazonie péruvienne
}

\author{
CÉLINE VALADEAU
}

Instituto Francés de Estudios Andinos (UMIFRE 17-MAE/CNRS-USR 3337)

et Centre EREA du LESC (UMR 7186)

Les Yanesha vivent sur les bas flancs des Andes. Leur territoire sétend des hautes aux basses terres de l'Amazonie Centrale péruvienne. Ces chasseurscueilleurs vivent principalement aux abords des fleuves Palcazu et Chanchamayo, dans la Région de Pasco, où ils y pratiquent une agriculture sur brûlis. Dans la mythologie yanesha et selon des conceptions fréquemment retrouvées dans la littérature orale des populations d'Amazonie, l'ensemble des êtres habitant la terre avait initialement forme humaine. Ces temps ontologiques se sont terminés par un moment crucial : celui de la spéciation. À ce moment-là, certains vivants changèrent d'apparence. C'est alors que des plantes, des animaux, des roches et de nombreuses entités non-visibles sont apparus. Selon des principes animistes, tous ces êtres vivants possèdent une intériorité - ou principe vital - de même nature mais se perçoivent sous des extériorités distinctes, c'està-dire des corps différents (Descola, 183-184). Ces transformations physiques ont été engendrées par la divinité créatrice Yato' Yosen en guise de punition envers les Humains qui lui avaient désobéi lors de son ascension vers les cieux. Ce châtiment s'est accompagné de l'apparition de la faim, de la maladie et de la mort, qui jusqu'alors n'existaient pas.

Ce bref aperçu de la mythologie yanesha permet de présenter la question qui est au centre de cet article et qui consiste à s'interroger sur les représentations 
que les Yanesha ont de l'environnement. Dans ce texte, je désire aborder cette question à travers la notion de ressource végétale utilisée lors des soins en utilisant les données issues de longs séjours sur le terrain.

Ce travail a débuté en 2006. Son objectif était de procéder à l'écriture d'une littérature orale : celle des plantes utilisées en thérapeutique (Valadeau et al. 2014, à paraître) $)^{1}$. Les relevés botaniques ont suivi la méthodologie classique $\mathrm{du}$ "relevé au pied de la plante $»^{2}$. Plus de 1500 planches botaniques furent enregistrées et déposées à l'herbier HOXA du Missouri Botanical Garden et à l'herbier national de Lima. Parallèlement à cette étude ethnobotanique, il s'est déroulé un travail ethnographique. Ainsi, aux relevés d'informations ethnobotaniques se sont ajoutés de nombreux matériaux ethnographiques obtenus grâce à des méthodologies conventionnelles telles que l'observation participante et l'étude des différentes formes de discours (cosmologie, anecdotes, discours chamaniques, formes rituelles, etc.). Le travail anthropologique a ensuite reposé sur l'analyse des informations ethnobotaniques et les matériaux ethnographiques.

Les paragraphes suivants questionnent la notion d'usage des plantes lorsque ces dernières entrent dans l'espace catégoriel des ressources. Je désire montrer à travers ces lignes que le végétal est une ressource vitale qu'il est nécessaire de considérer à la fois telle une subsistance, une dotation et un atout. En effet, tout au long de son existence, l'apport végétal revêt une importance cruciale. Il permet la formation du fotus et la croissance de l'enfant. Il prend également part à la création du caractère humain des jeunes adultes lors des rituels de passage. Enfin, il intervient également dans le processus final de transformation de l'humain incarné par la vieillesse. L'exposé s'organisera en fonction de ces trois étapes du cycle de vie afin de repenser l'univers végétal tel un élément constitutionnel de lêtre humain.

Afin d'introduire mon propos, j'évoquerai une citation relevée lors de ma présence sur le terrain. Elle dit : «les femmes comme toi nont pas d'enfants aussi jeunes que nous, là-bas ( « chez vous, en Occident ») il y a trop de béton. Cela ne fonctionne pas ». Cette affirmation étonnante amène la question de la nature des liens qui existent entre la capacité de reproduction de l'humain et la présence d'un milieu hostile caractérisé par l'absence de végétaux et d'animaux.

1. Dans un premier temps, ce document fut écrit et publié en espagnol. Destiné aux Yanesha, il a été déposé à INDECOPI (El Instituto Nacional de Defensa de la Competencia y de la Protección de la Propiedad Intelectual). Lien permanent : http://www. documentation.ird.fr/hor/fdi:010047903.

2. Permis de collecte $n^{\circ} 102 \_2007-I N R E N A-I F F S-D C B$, délivré par le Ministère de l'Environnement péruvien. 


\section{Du fœetus à l'enfant}

Des mythes racontent comment les plantes sont venues au monde sous la forme végétale que nous connaissons aujourd'hui, et comment certaines d'entre elles s'utilisent :

Une femme donna naissance à un enfant au bout de seulement quatre mois. Mais seules, la tête et la figure de l'enfant étaient formées, le reste nétait que du sang. Cette femme se sépara de cette difformité sanguinolente dans la forêt. Elle l'abandonna dans un cours d'eau. L'enfant mort-né était une petite fille. Peu de temps après, elle apparut à sa mère et lui dit: "Maman, je suis ta fille, celle que tu as laissée là-bas dans la forêt... Regarde-moi! À l'endroit même du sang que je répands sur le sol, vont désormais pousser des plantes. Elles se nomment sapeñtsopar ${ }^{3}$. Elles serviront à soigner les hémorragies auxquelles les femmes sont sujettes. Maintenant que tu connais ces plantes, montre-les aux autres femmes afin quielles aussi puissent soigner ces maux. ${ }^{4}$

Une version plus longue de cette histoire raconte que cette enfant mort-née s'est transformée, après son abandon, en entité nosologique. Elle est aujourd'hui dénommée Sapeñets et habite le monde non-visible.

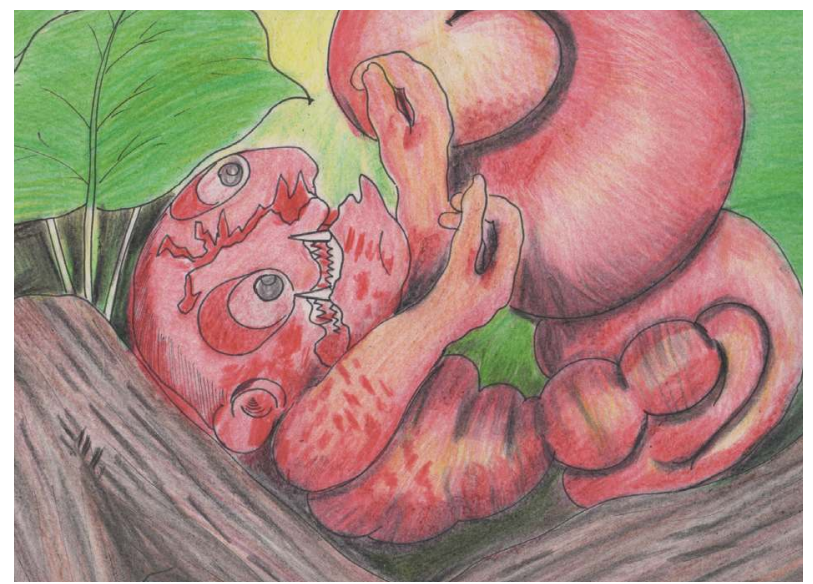

Illustration I - Représentation de l'entité nosologique nommée Sapeñets (dessin sur papier $21 \times 29 \mathrm{~cm}$ ) d'après le professeur bilingue Yanesha-Espagnol Anselmo Cruz Mariño.

3. Détermination botanique des sapeñtsopar : Geogenanthus rhizanthus (Ule) G. Brückn. (Commelinaceae) et Ruellia puri (Nees) Mart. ex B.D. Jacks (Acanthaceae). Dans certains cas de figure, une terminologie végétale yanesha sest avérée être définie par plusieurs dénominations latines. Note des éditeurs pour la lecture : nom d’espèce et, éventuellement, nom de genre suivi de nom(s) d’auteur(s) abrégé(s), entre parenthèse le nom de la famille de plantes.

4. Voir Valadeau, 2010: 122. 
Les entités nosologiques sont des êtres vivant sur terre dans le monde non-visible et reconnus comme pathogènes pour tout être humain. Seules les chamans, ou d'autres personnes en ayant l'habileté, ont la capacité de les entrevoir ; pour les autres, elles ne sont visibles qu'au travers de signes auditifs ou olfactifs. Sapeñets, elle, réside aujourd'hui dans les petits ruisseaux d'écoulement des forêts, aux abords desquels il est parfois possible de l'entendre pleurer. Sa présence est dangereuse pour les femmes enceintes. Comme toutes les entités nosologiques, elle consomme les principes vitaux et provoquent des fausses couches.

La fabrication du foetus est apparentée à une réaction produite à partir d'une quantité emmagasinée de sang féminin et d'une quantité accumulée de sperme. La cavité organique du sexe féminin sert ainsi de contenant. Le mélange des deux substances s'y fait et produit le fotus. C'est alors l'aménorrhée qui atteste de son existence (Valadeau 2012a, 61). Pendant cette période, la croissance intra-utérine est permise grâce à l'apport régulier, tout au long de la grossesse, des deux éléments originellement constituants : le sang et le sperme.

Dès le stade foetal, il est possible d'agir sur le devenir de cet être. Il est encore envisagé comme non-humain. Des plantes permettent tout d'abord de déterminer le sexe du futur nouveau-né. Tsanánarropan en est une ${ }^{5}$. Elle possède à la fois des feuilles de petite taille et de grande taille, alternées sur la même tige. Les feuilles les plus grandes sont consommées crues afin de mettre au monde un garçon. Les plus petites permettent donc de concevoir une fille. La femme enceinte doit aller, seule, choisir la feuille quelle va consommer et faire l'oraison appropriée. Lors de la grossesse, il est recommandé de réitérer cette démarche plusieurs fois. Pour s'assurer de l'efficacité de l'acte, chellochellpan est utilisée. Il s'agit en fait de deux plantes : Cyclanthusindivisus R. E. Schult. et Cyclanthusbipartitus Poit. ex A. Rich. (Cyclanthaceae). La première a les feuilles bien larges et non découpées, elle est désignée comme "féminine » et $C$. bipartitus possède des feuilles plus étroites, fendues en deux, elle est considérée comme " masculine ». La femme doit ouvrir un bouton floral de l'une des espèces. Si, une fois ouverte, l'apex présente une rayure transversale, l'enfant sera une fille. Ces plantes sont donc indicatrices, elles fournissent l'information convoitée. Les Yanesha considèrent que ces plantes, qui sont dotés d'une capacité communicationnelle, possèdent également un principe vital de nature ontologique identique à celui des Humains. Ainsi, par le biais d'une oraison, elles leur répondent par un signe tout à fait significatif.

Le genre n'a donc pas de relation directe avec le caractère humain. Constitutionnellement, tout être est composé d'un corps animé par un principe vital,

5. Détermination botanique : Pilea diversifolia Wedd. (Urticaceae). Dorénavant, indications entre crochets dans le corps du texte. 
yecamquëm, qui lui confère son intentionnalité (Smith, 96). Telle une aura, il se positionne tout autour du corps et grâce à un pouvoir d'adhésion, il diffuse dans la peau. De plus, le corps projette sur le sol une ombre, yechoyeshe'm. Sorte de fantôme, l'ombre est considérée comme constitutive de la personne et elle est caractérisée par sa capacité agentive. Le principe vital apparaît au stade foetal, au moment où la future mère ressent des mouvements dans son ventre. Ce principe vital provient de ceux contenus dans le sang maternel et dans le sperme. En effet, toutes les substances corporelles ayant pour dessein dêtre sécrétées ou excrétées sont " chargées » d'une partie du principe vital de la personne. Le sperme est considéré comme hautement chargé en principe actif masculin et toujours conçu comme très puissant. Dès la naissance, l'administration du végétal devient essentielle. Le nouveau-né non encore humain est fragile. Il doit faire l'objet de nombreux soins : eñeserch [Clusia amazonica et Clusia hammaeliana (Clusiaceae)] permettent d'augmenter le volume de principe vital $\mathrm{du}$ foetus in utero, puis du nourrisson afin qu'il grandisse bien, s'exprime clairement et travaille avec entrain. Hormis les plantes utilisées afin de garder ce petit corps en vie, d'autres plantes sont nécessaires et permettent de construire son tempérament : huomencpar [Cyperus sp. (Cyperaceae)] ${ }^{6}$ s'administre afin que le futur adulte soit courageux ; tsenantsopar [Costus conicus Stokes (Zingiberaceae)], pour qu'il soit obéissant et qu'il n'ait jamais de mauvaises pensées ${ }^{7}$. À cet égard, les exemples de plantes à usages " comportementaux » sont nombreux. Les connaissances de chaque famille sont différentes et cela explique la diversité des tempéraments. Des remèdes animaux peuvent aussi être utilisés tels que tot, une grande chenille verte (Trichoplusiani) que

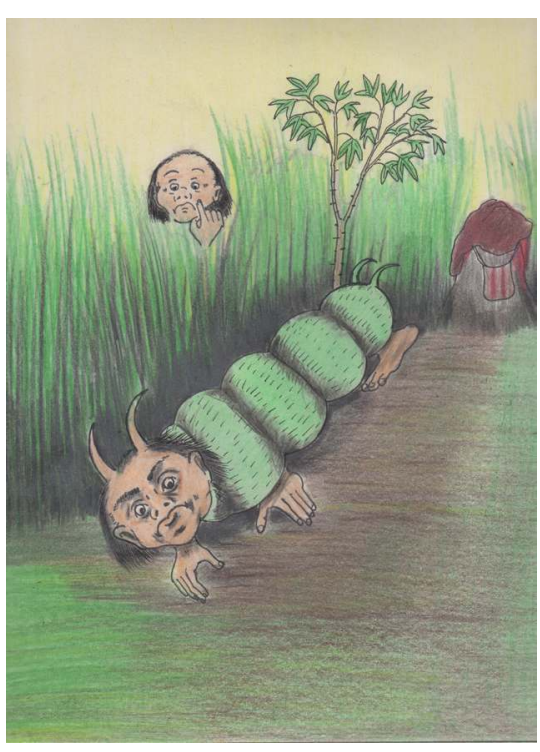

Illustration II - Dessin sur papier de la chenille Totana'. Dans la mythologie cette femme avait été envoyée sur terre pour aider les Yanesha à s'accoutumer à leur nouvelle existence mortelle. Elle travaillait très rapidement dans les abattis. Un jour, une femme en surpris son activité et devina qu'elle n'était pas humaine. Depuis ce jour, Totana' est une grande chenille verte que I'on retrouve dans les abattis sur les plants de manioc. Anselmo Cruz Mariño, $21 \times 29 \mathrm{~cm}$.

6. Note des éditeurs : «sp. » est l'abréviation de species (espèces). Elle indique qu'il s’agit d'un genre englobant de nombreuses espèces.

7. Lorsqu'elles sont exprimées, les mauvaises pensées sont potentiellement nocives pour la personne envers qui elles sont dirigées. Il s’agit d'une sorte de sorcellerie secondaire commune. 
les jeunes filles frottent entre leurs mains régulièrement afin d'être vaillantes et de travailler rapidement dans les abattis.

Ainsi, les Yanesha utilisent des plantes dès la période foetale. Laction vise le principe vital, en d'autres termes, elle cherche à garantir la vitalité de la matière organique foetale.

Après la venue au monde, les soins végétaux sont renouvelés de nombreuses fois. Toutes ces plantes sont cueillies et administrées dans le but de construire d'une part, lêtre vivant et d'autre part, la personne sociale. Dans ce contexte, l'action des plantes possède comme fondement la capacité des principes vitaux à interagir entre eux. Intrinsèquement identiques, ils peuvent s'agréger, induisant alors des phénomènes de transformations. Il s'agit, alors, de transformer progressivement le petit être nouvellement né afin qu'il puisse, dans un premier temps, marcher, parler, puis comprendre et apprendre. Ces soins s'apparentent donc à un processus de socialisation par les plantes (Rama-Leclerc, 435). L'administration de leurs principes vitaux permet, ainsi, une pleine intégration parmi les Humains.

\section{Passage à l'âge adulte et humanité acquise}

Tout au long de l'enfance, l'enfant est soigné avec diverses plantes afin de renforcer tel ou tel comportement. Dès qu'il manifeste un caractère particulier, un prénom lui est attribué. À ce moment-là, l’enfant est désormais identifiable par son nom. S'il vient à décéder, la famille procède à de réels rituels funéraires. C’est au moment de la puberté que se joue la dernière étape de construction de l'humain. Elle signe le passage vers une humanité considérée comme entière. Cette étape est particulièrement importante et beaucoup plus ritualisée pour les jeunes filles. L'apparition de leurs premières menstruations est le signe du commencement du rituel (Santos-Granero, 272). La jeune enfant est alors protégée pendant quelques semaines à l'intérieur d'une petite hutte faite de feuilles de palme tressées. Elle doit y séjourner et y reçoit les enseignements et les soins de ses ainées. Ses cheveux sont coupés et remplacés par un long voile noir. Ainsi débarrassée des empreintes de l'enfance, l'administration des remèdes végétaux permet leur renouvellement. Les plantes Echtallets [Xiphidium caeruleum Aubl.] et c̈hap [Sida rhombifolia L.] sont utilisées sous forme de bain, elles servent à fortifier les cheveux afin qu'ils poussent vite, de couleur noire comme l'ébène et qu'ils ne tombent pas. Son tempérament est aussi affirmé par des plantes telles que pesharrpar [Strychnos toxifera R.H. Schomb], plante d’odeur agréable, elle est mâchée et prédispose à bien cuisiner ; puensenpar [Piper sp.] et epe' huomnecpar [Cyperus sp.] permettent de donner du courage, de ne pas être paresseuse et d'accomplir les tâches domestiques rapidement. La construction 
de sa personne s'achève à sa sortie de la hutte, cela signe la rupture du rituel de passage.

Entrée dans lâge adulte, la jeune fille n'a plus besoin de protection parentale. Le principe vital des parents ne la protège plus car le sien est considéré comme suffisant. Toutefois, cela ne signifie pas que l'administration de plantes s'arrête là. Le caractère humain est instable. En effet, comme le principe vital ne reste pas confiné, il peut être enlevé ou modifié par une entité nosologique ou perdu dans les sécrétions et excrétions quotidiennes. Ces substances organiques perdues peuvent être remplacées à l'aide de remèdes. Par exemple, les larmes perdues sont compensées par une préparation faite à partir de tubercules de mamrë̆, [Martinella obovata (Bignoniaceae)]. Le végétal est finement haché afin dêtre enveloppé dans un linge qui, par pression,

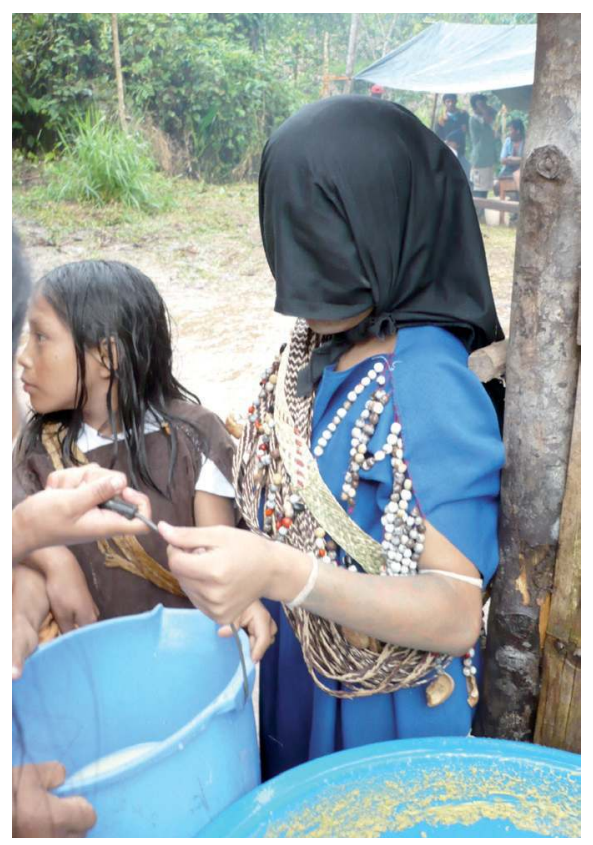

Photo 1 - Jeune fille lors de la cérémonie clôturant son rituel de passage dans la communauté yanesha de Santa Rosa de Pichanaz. Elle reçoit ses invités en leur offrant de la bière de manioc. Photo : Céline Valadeau. permet l'extraction du jus destiné à être instillé dans l’œil. De même, la salive est régénérée en coupant un tronçon de liane d'une cinquantaine de centimètres de ma'yarrosheshep [Uncariaguianensis (Aubl.) J.-F. Gmel (Rubiaceae)], «l'eau » qui s'en écoule est bue jusqu'à ce que le bois en soit épuisé. Pour sa part, che'llcapan [Desmodium adscendens (Sw.) DC. (Fabaceae)] permet de restaurer l'urine excrétée : les racines de cette plante sont préparées en décoction. La plante ho'ne'ch [Gynerium sagittatum (Aubl.) P. Beauv. (Poaceae)], dont les jeunes pousses sont également bues sous forme de décoction, permet la production de sperme. Dans le cas d'une perte de sang, quelle soit due aux menstruations ou à d'autres étiologies ${ }^{8}$ appartenant à la nosologie, et comme le mythe l'évoque, des sapeñtsopar peuvent être préconisées. Ces plantes se préparent souvent ensemble, mais peuvent aussi être utilisées séparément. Une vingtaine de feuilles, cueillies cinq par cinq sur des rameaux différents, sont mises à bouillir dans de l'eau. Lorsque la préparation est bien rouge, un demi-verre est bu trois fois par jour jusquà ce que l'hémorragie cesse. La couleur et l’odeur de la préparation

8. Dans la nosologie yanesha, les étiologies courantes sont les attaques des entités nosologiques et les actes de sorcellerie. 
attestent du passage du principe vital de la plante dans l'eau. Comme dans de nombreuses autres préparations, il s'agit d'un processus de création de la forme ontologique. La décoction rouge est, en définitive, le sang perdu par Sapeñets, le sang qui donna naissance à cette plante. Ce sang permet à l'Humain de reconstruire son propre sang et d'en retrouver le volume corporellement perdu (Valadeau 2010, 227).

Ces préparations de plantes permettent, donc, la reconstruction du caractère humain de la personne, qui a tendance à s'estomper un peu chaque jour. Le principe vital et les substances corporelles sont agrémentés lorsque le besoin s'en fait sentir comme lors d'un état de faiblesse ou d'une maladie. Le reste du temps, l'alimentation joue ce même rôle, mais d'une manière moins spécifique. La personne est ainsi continuellement dans un processus de transformation à la frontière de l'humain et du non-humain, au cours duquel le végétal est une ressource indispensable au maintien de son caractère humain.

\section{Transformations finales de l'humain}

Une période de la vie correspond à la vieillesse. Elle commence avec l'apparition des rides, des cheveux blancs et de certaines douleurs articulaires. La vieillesse annonce une transformation avancée de la personne et son départ vers d’autres formes de vivants ${ }^{9}$. Des écrits mentionnent que la mort est généralement provoquée par une étiologie externe à l'humain (Zempléni, 20). Or, chez les Yanesha, il semble qu'il existe un phénomène de "mort naturelle ». Tout comme le décrit Jean Chapuis chez les Wayana, il s'agit d'une transformation qui est provoquée par une inadéquation progressive du principe vital (Chapuis, 43). Cette tendance implique un facteur principal : celui de la sécheresse corporelle qui apparait au cours du temps. Ce changement d'aspect cutané, identifié sous forme de rides, est principalement dû à la présence de sel à la surface de la peau. En effet, en Amazonie, le sel possède un caractère ambivalent (LéviStrauss, 252). Hormis le fait qu'il soit vital, il est capable d'assécher la matière et de la priver de vitalité. Ce phénomène est souvent constaté lorsque la viande est salée en vue de sa conservation. Les explications données à cet égard prennent en compte le principe vital de la matière jusqu'alors vivante. La fonction du sel est de faire sortir le principe vital initialement diffusé dans son support, ce qui le prive, alors, de toute animation ou vie. Ainsi, le sel, en étant excrété sur la peau, joue ce rôle : il a tendance à extraire le principe vital diffusé dans la peau et l'assèche. Or, cette capacité que possède le sel trouve aussi un sens lorsqu'il

9. Il existe plusieurs formes dêtres vivants. Dans la cosmologie yanesha, il existe plusieurs mondes ou terres superposées. Parmi elles, certaines hébergent les êtres défunts. Ainsi, les victimes d’assassinats vivent indépendamment des morts de vieillesse. 
est question de l'administration des remèdes. En effet, ceux-ci sont préparés dans le but de présenter au corps un principe vital végétal ou animal dans le but dêtre associé à la personne. L'administration du remède permet au principe vital de s'engager sur des voies de circulations intracorporelles, chacune d'entreselles définies par un procédé de soin, une voie d'administration et un processus d'assimilation (Valadeau, 2015). Par exemple, le contact initial peut être fait à l'aide de la respiration, comme dans le cas des bains de vapeur, de la peau lors d’application cutanée, ou encore par voie orale lorsque le remède est ingéré.

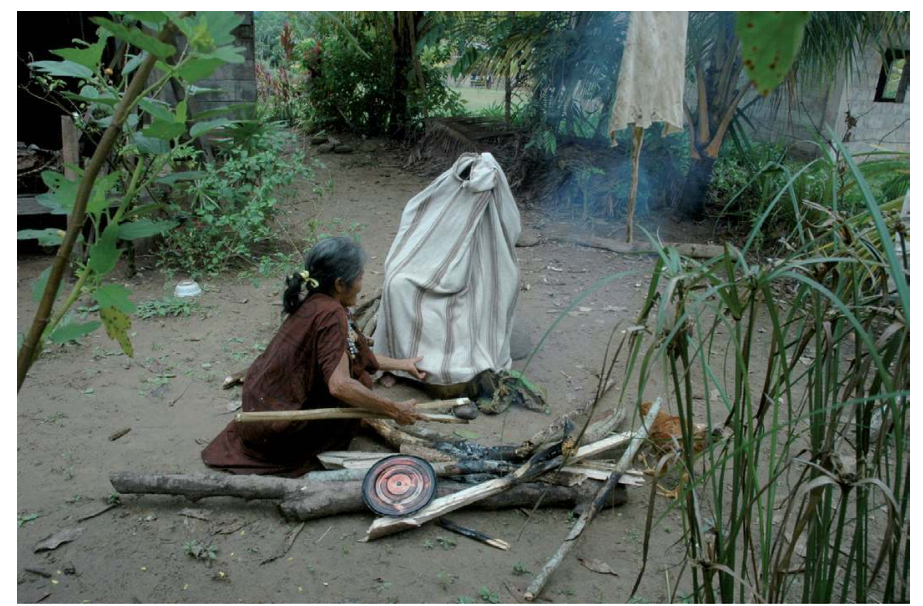

Photo 2 - Procédé de soin par bain de vapeur, une petite enceinte close de sudation. Ce soin est toujours assisté d'une personne qui agrémente le récipient contenant le remède en pierres chaudes afin d'engendrer une ébullition et donc une évaporation plus intense. Photo : Céline Valadeau.

Ces remèdes sont tels des extraits de principes vitaux ; lorsqu'ils sont contenus dans le corps, ils y circulent. Ces circulations sont permises grâce au sel qui les transporte à la surface de la peau par le phénomène de transpiration. Une fois sur la peau, le sel aide encore à leur évaporation. Une fois revenu à létat nontangible, les principes vitaux végétaux s'agrègent à celui de la personne et le complémentent.

De par cette inadéquation progressive, les personnes âgées sont également plus vulnérables, leur principe vital est plus faible. Elles sont donc plus enclines à être attaquées par des entités nosologiques. Dans tous les cas, elles sont dans un processus de transformation qui les amène à devenir autres, à changer de corps afin d'intégrer le monde terrestre nonvisible. Ainsi, si leur décès est provoqué par des entités nosologiques, le «fantôme » de la personne défunte, c'està-dire son ombre, prendra la forme de celles-ci afin d'en rejoindre le groupe dans le monde nonvisible. Dans le cas où la mort est naturellement engendrée 
par le sel, l'ombre de la personne va trouver sa place sur la terre des choyeshe'mats ou "fantômes naturels » qui se situe au-dessous de la terre de résidence des Humains.

Ainsi, la mort constitue, aussi, un phénomène de transformation (Taylor, 41). Elle peut être engendrée par le sel comme par la maladie ${ }^{10}$. Il s'agit d'un passage du monde humain tangible au monde nonhumain nontangible. Il y a là une continuité de lêtre par changement de formes. Cette autre apparence place alors la personne devenue autre, dans un groupe social différent, celui des entités nosologiques par exemple. En un sens, la mort constitue un moyen d'alimenter le monde nonvisible en êtres lui correspondant, selon l'idée qui se rapprocherait de celle d'une sorte de cycle des vivants.

\section{Quelle continuité entre les vivants ?}

Le caractère humain de la personne est donc en continuel mouvement et peut être perturbé à tout moment. Ainsi, la citation yanesha, mettant en corrélation la stérilité féminine et l'absence de végétaux, s’éclaire désormais. Elle fait référence à l'absence de végétal et à son caractère indispensable dans la construction et le maintien de l'humanité de la personne. Dès lors, la notion de ressource thérapeutique ou alimentaire amène très clairement à celle de subsistance. Ces plantes sont essentielles, autant à la vie, car sources de principes vitaux indispensables, qu'au bon fonctionnement social, puisqu'elles permettent l'acquisition des comportements nécessaires pour s'y intégrer. L'essence des plantes ou des animaux est donc bien essentielle et entre pleinement dans la composition et dans le fonctionnement de l'humain. Ainsi, entre l'humain et le végétal ou l'animal, la dualité n'existe pas. La personne est perpétuellement prise dans un processus continu de "devenir autre " grâce à un processus de partage des substances (Rival, 106). Les identités sont, en un sens, ponctuelles. Alors, pour reprendre les dires de Tim Ingold, ce que constitue l'environnement, pris au sens de milieu, ne peut être considéré comme un ensemble sopposant à l'humain. L'environnement constitue un tout fonctionnel, il se compose de tous les éléments qui s'y trouvent, y compris l'Homme (Ingold, 180).

Pour finir, qu'est-il alors possible d'envisager lorsqu'il est question de ressource thérapeutique ou naturelle ? Dans ce contexte, la notion de ressource naturelle suit l'idée de matière première indispensable à la vie humaine, autant en terme constitutionnel que fonctionnel, mais elle prend aussi une autre dimension. En effet, si l'humain en éprouve un besoin incontestable, l'inverse est

10. La personne affectée par une entité nosologique ou par des actes de sorcellerie, mentionne des symptômes. 
aussi vrai puisque la production d'humain ne se limite pas à une nécessité reproductive. Au sein de ce tout fonctionnel, la plante intègre l'humain, l'humain se change en nonhumain et le nonhumain peut prendre des formes humaines. Ainsi, ce qui appartient au visible passe volontiers dans le monde nonvisible par transformation et intégration des substances et inversement. Dans cette région d'Amazonie, il est alors difficile, voire impossible, de séparer les différents éléments du monde. L'idée de ressource implicite la notion de matériel indispensable à lêtre humain. Or, cette dualité entre humains et nonhumains ne trouve pas de pertinence ici, les ressources doivent être pensées comme des éléments potentiellement transformables qui entrent dans la dynamique des vivants. Il existe, ici, une réelle continuité entre les êtres vivants isolement identifiables et cette continuité possède comme essence la capacité transformationnelle des êtres par le jeu de « devenir substance ». Tous les êtres sont en transformation et ils sont utiles les uns aux autres, autant dans leur fonctionnalité que dans leur constitution, telle une condition d'existence et d'incarnation. Tous sont un ; ils sont autant ressources pour les uns que pour les autres.

\section{Bibliographie}

CHAPUIS, Jean, La personne wayana entre sang et ciel, Thèse de doctorat, Université Aix-Marseille, 1998, 284 p.

DESCOLA, Philippe, Par-delà nature et culture, Paris, Gallimard, coll. « Bibliothèque des Sciences Humaines ", 2005, 624 p.

INGOLD, Tim, "Culture, nature et environnement ", Tracés - Revue de Sciences humaines 22, (2012), disponible sur : http://traces.revues.org/5470 ; DOI : 10.4000/traces.5470, consulté le 18 décembre 2013.

LÉVI-STRAUSS, Claude, Du Miel aux Cendres, Paris, Plon, 1966, 450p.

RAMA-LECLERC, Frédérique, Des modes de socialisation par les plantes chez les Shipibo-Conibo d'Amazonie péruvienne, une étude des relations entre humains et non-humains dans la construction sociale, Thèse de Doctorat, Université Paris X - Nanterre, 2003, 555 p.

RIVAL, Laura, "What Constitutes a Human Body in Native Amazonia? Introduction to the Special Issue of Tipití on the Amazonian Body." Tipití 3.2, 2005: 105-110.

SANTOS-GRANERO, Fernando, "Los Yanesha ", dans F. Santos-Granero et F. Barclay, Guía etnográfica de la Alta Amazonía (volumen 5), Paris-Balboa, Institut Français d'Études Andines \& Smithsonian Tropical Research Institute, 2004, 158-368. 
SMITH, Richard Chase, Delivrance from Chaos for a Song: a Social and Religious Interpretation of the Ritual Performance of Amuesha Music, Ph.D., Graduate School of Cornell University, 1977, 326 p.

TAYLOR, Anne-Christine, «Le corps de lâme et ses états - Être et mourir en Amazonie », Terrain, 52 (2009), 39-49.

VALADEAU, Céline, De l'ethnobotanique à l'articulation du soin: Approche du système nosologique chez les Yanesha de Haute Amazonie péruvienne, Thèse de Doctorat, Université Paul Sabatier-Toulouse, 2010, 382 p.

, La traversée par les plantes: Médecine chez les Yanesha de Haute Amazonie péruvienne, Paris, L'Harmattan, 2012a, 388p.

"Catégorisation des plantes et des entités étiologiques chez les Yanesha (piémont amazonien du Pérou) », Bulletin de l'IFEA 41.2 (2012b), 241281.

«Des substances en mouvement : le rôle du sel dans le soin des semi-morts chez les Yanesha (Haute Amazonie péruvienne) », Journal de la Société des Américanistes, 2014 (en cours de publication).

VALADEAU, Céline et Geneviève BOURDY, Lesprit de la plante, le corps de l'homme : approche logique du monde végétal Yanesha, Montpellier, Éditions IRD, 2014 (sous presse), $350 \mathrm{p}$.

ZEMPLÉNI, Andréa, "La "maladie" et ses "causes". Introduction ", Lethnographie, numéro spécial "Causes, origines et agents de la maladie chez les peuples sans écriture », 96-97.2-3 (1985), 13-44.

Résumé : Dans un contexte amazonien, est-il possible de parler de « ressources thérapeutiques »? Cet article propose de s'intéresser à la notion de ressources médicinales chez les Yanesha du Piémont péruvien. Les travaux ethnographiques, ethnobotaniques et anthropologiques effectués permettent de définir avec précision la nature des représentations des matières végétales et animales lorsque celles-ci sont cherchées, transformées et utilisées à des fins thérapeutiques. Ce texte propose de mettre en perspective les différentes représentations du vivant avec les notions de la personne Yanesha et ce, dans le but d'examiner, d'un point de vue ontologique et fonctionnel, comment se conçoivent les frontières entre personne et ressource. En somme, il s'agit de réfléchir, à travers les différentes formes de thérapeutiques utilisées, à la question de continuité et de discontinuité du vivant.

Mots-clés : plante, ressource, médecine, corporéité, Piémont amazonien, Pérou, Yanesha.

Resumen: En contexto amazónico, ¿podemos hablar de "recursos terapéuticos"? Este artículo quiere presentar una reflexión sobre lo que representa el concepto de recursos entre los Yanesha de la ceja de selva peruana. Los estudios etnográficos, antropológicos e etnobotánicos realizados permiten describir con precisión las representaciones asociadas a la materia vegetal y animal cuando estas últimas son utilizadas como terapéutica. Se pretende poner en perspectiva las representaciones del ser vivo con el concepto de la persona Yanesha, con el fin de comprender, desde un punto de vista ontológico y funcional, cuáles son las fronteras entre la persona y lo que se llama recurso. Así la propuesta es la de examinar, a través de las diferentes terapéuticas utilizadas, la cuestión de continuidad y de discontinuidad entre los seres. 
Palabras clave: Planta, recurso, medicina, corporeidad, Alta Amazonía, Perú, Yanesha.

Céline Valadeau est chercheur pensionnaire à I'Institut Français d'Etudes Andines en Amérique Latine (UMIFRE 17-MAE/CNRS-USR 3337) et associée du Centre d'Enseignement et de Recherche en Ethnologie Amérindienne (EREA) du Laboratoire d'Ethnologie et de Sociologie Comparée (LESC, UMR 7186). Anthropologue et ethnobotaniste, ses travaux se sont principalement effectués en Amazonie et plus particulièrement au Pérou puis en Colombie. Elle s'interroge essentiellement sur les principes de corporéité parmi lesquels, ceux associés à l'univers végétal au sein des populations Yanesha et Cubeo.

Céline Valadeau es investigadora del Instituto Francés de Estudios Andinos (UMIFRE 17-MAE/CNRSUSR 3337) y asociada en el Centro de Enseñanza e Investigación en Etnología Amerindia (EREA) del Laboratorio de Etnología y de Sociología Comparada (LESC, UMR 7186). Antropóloga y etnobotánica, sus trabajos han sido principalmente llevados a cabo en Amazonia y más precisamente en Perú y Colombia. Su investigación se centra fundamentalmente en los principios de corporeidad, entre los cuales, los que se relacionan con el mundo vegetal en la cultura de los pueblos Yanesha y Cubeo. 